\title{
Guest Editorial FPL 2013
}

The International Conference on Field Programmable Logic and Applications (FPL) is one of the most prestigious research and development events in the area of fieldprogrammable logic. The FPL conference series aims at bringing together researchers and practitioners from both academia and industry worldwide for broad discussions and exchanges on the theoretical and practical issues facing this rapidly evolving area, including, but not limited to applications, advanced Electronic Design Automation (EDA), novel system architectures, embedded processors, arithmetic, and dynamic reconfiguration. The 23rd FPL continued the tradition of previous FPL editions and took place on September 2-4, 2013, in Porto, Portugal.

It is our pleasure to present in this special ACM TRETS issue extended versions of a selected set of five papers accepted and presented at FPL 2013. The articles included in this special issue and their research contributions and highlights, which we present below, have been selected out of the 57 conference papers covering various topics, namely CAD (Kevin E. Murray et al., and Ricardo Ferreira et al.), communication security (Zsolt István et al.), fault-tolerance (Anup Das et al.), and scientific computations (Lin Gan et al.).

The article entitled "Timing-Driven Titan: Enabling Large Benchmarks and Exploring the Gap between Academic and Commercial CAD," by Kevin E. Murray, Scott Whitty, Suya Liu, Jason Luu, and Vaughn Betz, presents a framework and representative complex benchmarks to evaluate mapping, placement, and routing algorithms for island-style FPGA architectures. The framework uses VPR and allows the use of large benchmarks and the exploration of algorithms when targeting an enhanced version of the Altera's Stratix IV FPGA architecture. By providing a comparison of the results achieved by VPR with those achieved by Altera's Quartus II CAD software, this work reveals its high importance for further scientific research related to mapping, placement, and routing.

The article entitled "A Runtime FPGA Placement and Routing Using Low Complexity Graph Traversal,” by Ricardo Ferreira, Luciana Rocha, André Santos, José Nacif, Stephan Wong, and Luigi Carro, proposes a placement and routing algorithm for homogeneous FPGAs that is suitable for use at runtime. The algorithm is based on a graph mapping approach and uses a greedy polynomial time heuristic. Compared to stateof-the-art approaches, the algorithm is able to place and route designs very quickly, without significantly compromising placement and routing quality. Due to the growing importance of runtime placement and routing, this article is a step forward on researching efficient algorithms to make it practical.

The domain of Internet communication for simple routing to security is a growing area of applicability for FPGA technology. In this context, the article "A Hash Table for Line Rate Data Processing," by Zsolt István, Gustavo Alonso, Michaela Blott, and Kees Vissers, proposes and evaluates a novel multistage data-flow architecture for the use of key-value stores. Their solution is very impressive, supporting a broad range of key sizes without stalling internal pipelining while still being parameterizable to work with different memory types operating at different access speeds and latencies. The proposed solution can index 1 million entries in 24GB of external DDR3 DRAM while sustaining 13 million requests per second UDP binary encoded mem-cached packets.

(c) 2015 ACM 1936-7406/2015/03-ART8 $\$ 15.00$

DOI: http://dx.doi.org/10.1145/2737805 
The article entitled "Autonomous Soft-Error Tolerance of FPGA Configuration Bits," by Anup Das, Shyamsundar Venkataraman, and Akash Kumar, examines the idea of masking faults (SEUs in particular) in LUTs' configuration data by restructuring logic and exploiting unused logic circuitry within an FPGA. More resource-efficient than TMR, the approach makes use of only two copies of a (possibly modified) logic function, combining the results using AND or OR logic functions implemented in the FPGA's special carry-chain structures. The proposed Boolean transformations also make use of excess XOR gates to increase percentage of faults masked in each LUT. Combined, these techniques masked approximately $85 \%$ of injected faults in the LUTs of tested benchmarks with relatively low area overheads.

Last, the use of FPGA-based computing systems to speed up computations has long been recognized, in various instances even outperforming GPU-based solutions. The article entitled "Solving the Global Atmospheric Equations through Heterogeneous Reconfigurable Platforms," by Lin Gan, Haohuan Fu, Wayne Luk, Chao Yang, Wei Xue, and Guangwen Yang, describes the use of FPGAs to solve the problem of shallow water equations occurring in atmospheric physics. In this work, the authors demonstrated the use of mixed-precision arithmetic to implement a hybrid CPU-FPGA design yielding substantial performance improvement over traditional multicore solutions-and even over a hybrid contemporary CPU-GPU system.

As guest editors, we would like to thank all authors for the high quality of their work and the anonymous reviewers for their efforts in evaluating and suggesting improvements to these articles. We also would like to acknowledge the support of Wayne Luk (previous ACM TRETS Editor-in-Chief), Steve Wilton (ACM TRETS Editor-inChief), Philip Leong (ACM TRETS Senior Associate Editor), and David Thomas (ACM TRETS Information Editor).

We hope you enjoy reading the articles included in this special issue and that they serve as useful sources of research inspiration,

João M. P. Cardoso

Universidade do Porto, Porto, Portugal (jmpc@acm.org)

Pedro C. Diniz

USC Information Sciences Institute, USA (pedro@isi.edu)

Katherine (Compton) Morrow

University of Wisconsin-Madison, Madison, USA (kati@engr.wisc.edu)

Guest Editors 